\title{
ON THE LIE-KOLCHIN-MAL'CEV THEOREM
}

\author{
B. A. F. WEHRFRITZ
}

(Received 18 August 1977)

\begin{abstract}
We discuss generalizations of the Lie-Kolchin-Mal'cev theorem. For example we show that if $G$ is a soluble linear group of degree $n$, then $G$ contains a triangularizable subgroup $T$ whose index in $G$ is bounded by function of $n$ only and such that $T$ is normalized by every automorphism of $G$ normalizing $G^{0}$, the Zariski connected component of $G$ containing the identity. We also prove that in certain situations at least the index of $G^{0}$ in $G$ can be bounded in terms of the degree and the ground field.
\end{abstract}

Subject classification (Amer. Math. Soc. (MOS) 1970): 20 H 20.

A soluble subgroup $G$ of $\mathrm{GL}(n, F)$, throughout $n$ denotes a positive integer and $F$, a commutative field, contains a triangularizable normal subgroup of finite index. There are essentially two approaches to this theorem. The Lie-Kolchin method (Wehrfritz (1973), 5.8) uses the Zariski topology to prove that the connected component $G^{0}$ of $G$ containing the identity is triangularizable. (All topological terms in this note refer to the Zariski topology.) Mal'cev's and related proofs (cf. Wehrfritz (1973), Chapter 3) produce a triangularizable normal subgroup $T$ of $G$ of finite index bounded by a function of $n$ only. It is elementary that one can also choose $T$ closed in $G$, so $T \supseteq G^{0}$, but one cannot in general choose $T$ connected without losing the bound on its index (just consider finite groups). In this note we investigate situations where this can, or very nearly can, be done (Corollaries to Proposition 2 below). We also prove a strengthening of Mal'cev's theorem that is universally true (Proposition 1 below).

$G^{0}$ is topologically characteristic in $G$, that is $G^{0}$ is normalized by every homeomorphic automorphism of $G$. Our first result says that we can choose the $T$ in Mal'cev's theorem to be closed and topologically characteristic in $G$, after increasing the bound somewhat. Since all homeomorphic automorphisms of $G$ normalize $G^{0}$ the following is more general. 
Proposition 1. Let $G$ be a soluble linear group of degree $n$. Then $G$ contains $a$ closed triangularizable subgroup $T$ normalized by all automorphisms of $G$ normalizing $G^{0}$ and such that the index $(G: T)$ is bounded by a function of $n$ only.

A suitable function of $n$ can be given explicitly. We make no attempt to find the best possible function. The characteristic zero case of Proposition 1 is very simple and in fact here we obtain a slightly stronger conclusion from the weaker hypothesis that $G$ is only soluble-by-finite, see Lemma 1 . It is very easy to produce soluble examples $G$ where we cannot find a characteristic triangularizable subgroup of finite index in $G$, for example, Wehrfritz (1978), Section 4.

COROLlaRY. Let $G$ be a subgroup of $\Gamma L(n, F)$ and $S$ a soluble normal subgroup of $G$ with $S \subseteq \mathrm{GL}(n, F)$. Then $S$ contains a triangularizable subgroup $T$ of finite index bounded in terms of $n$ only, such that $T$ is normal in $G$.

For every element of $\Gamma L(n, F)$ induces by conjugation a homeomorphism of $\operatorname{GL}(n, F)$. The special case of this corollary where $G$ too lies in $\operatorname{GL}(n, F)$ was first recorded, as far as I know, in Gruenberg (1968).

It is sometimes necessary to consider connected and not just topologically characteristic triangularizable subgroups, but to produce such subgroups of boundably finite index we need to restrict the field. If $F$ is any field define a map $\delta: \mathbf{N}^{+} \rightarrow \mathbf{N}^{+} \cup\{\infty\}$ by setting $n \delta$ equal to the least common multiple of the orders of all roots of unity in the splitting fields over $\boldsymbol{F}$ of polynomials over $\boldsymbol{F}$ of degree $n$. We call $\delta$ the depth function on $F$ and say $F$ is shallow if $n \delta$ is finite for every $n$. More generally, if $n \delta<\infty$ we say $F$ is shallow at $n$.

It is well known that the rational field and for each prime $p$ the field of $p$-adic numbers are shallow fields (for example Wehrfritz (1973), 9.33). It is trivial that every finite field is shallow. Further examples are provided by the following lemma, the first statement of which follows from the second since prime fields are shallow.

LEMMA 5. Every finitely generated field is shallow. More generally, so is every finite extension of a purely transcental extension of a shallow field.

Our second main result is the following.

Proposition 2. Let $G$ be a subgroup of $\operatorname{GL}(n, F)$ where the field $F$ is shallow at $n$. If $\operatorname{char} F=0$ then $G / G^{0}$ has exponent dividing $n \delta$ and order dividing $n !(n \delta)^{n}$. If char $F=p>0$ the $p^{\prime}$-elements of $G / G^{0}$ have order dividing $n \delta$ and the $p^{\prime}$-part of $\left(G: G^{0}\right)$ divides $n !(n \delta)^{n}$.

Notice that there is no solubility restriction on $G$ here. In view of Lemma 5, Proposition 2 generalizes, with a different bound, Wehrfritz (1978), 4.1, which 
treats by very different methods to those below the case of linear groups over finitely generated integral domains. The following is an immediate consequence of Proposition 2 and the Lie-Kolchin theorem.

COROLLARY 1. If $G$ is a soluble-by-finite subgroup of $\mathrm{GL}(n, F)$ where the field $F$ has characteristic 0 and is shallow at $n$, then $G^{0}$ is a closed connected triangularizable normal subgroup of $G$ of finite index bounded in terms of $n$ and $F$ only.

It is trivial that the conclusion of Corollary 1 above is false for any infinite field of positive characteristic. If $G \subseteq \mathrm{GL}(n, F)$ where char $F=p>0$, as in Wehrfritz (1978) define $G^{+}$by $G^{+} / G^{0}=O_{p}\left(G / G^{0}\right)$. The best we have obtained for fields of positive characteristic is the following.

COROLLARY 2. Let $G$ be a subgroup of $\mathrm{GL}(n, F)$ where the field $F$ has positive characteristic $p$ and is shallow at $n$. Then

(a) the index $\left(G: G^{+}\right)$divides $\left(n !(n \delta)^{n}\right)$ !,

(b) if $G$ is nilpotent then $G^{+}$is triangularizable and

(c) if $G$ is soluble-by-finite $T=C_{G+}\left(G^{0} / u\left(G^{0}\right)\right)$ is a triangularizable subgroup of $G$ such that $G^{0} \subseteq T, T$ is an extension of a connected group by a finite p-group, $N_{\text {Aut } G}\left(G^{0}\right)$ normalizes $T$ and $(G: T)$ divides $n ! .\left(n !(n \delta)^{n}\right)$ !.

Here $u(X)$ denotes the unipotent radical of the linear group $X$, that is, the unique maximal unipotent normal subgroup of $X$.

A slight extension of Mal'cev's theorem, due to Platonov, see Wehrfritz (1973), 10.11 , states that there exists an integer-valued function $\alpha$ such that every solubleby-finite linear group of degree $n$ and characteristic 0 has a triangularizable subgroup of finite index dividing $n \alpha$. We choose $\alpha$ so that $n \alpha$ is minimal for every positive integer $n$.

LeMMA 1. Let $G$ be a soluble-by-finite subgroup of $\mathrm{GL}(n, F)$ where $\operatorname{char} F=0$. Then $G$ contains a triangularizable subgroup $T$ such that $G^{0} \subseteq T, T / G^{0}$ is a characteristic subgroup of $G / G^{0}$ and $(G: T)$ divides $(n \alpha)^{n+1}$.

Proof. Clearly we may assume $F$ is algebraically closed. Denote the (Zariski) closure in $\mathrm{GL}(n, F)$ of a subset $X$ of GL $(n, F)$ by $\bar{X}$. It is easily seen that $\left(\bar{G}^{0}\right)=(\bar{G})^{0}$, $\bar{G}=G G^{0}$ and $G^{0}=G \cap \bar{G}^{0}$. Also by a lemma of Platonov (Wehrfritz (1973), 10.10) there exists a finite subgroup $H$ of $\bar{G}$ with $\bar{G}=H \bar{G}^{0}$.

Here $G^{0}$ and hence $\bar{G}^{0}$ is soluble, so there exists a triangularizable subgroup $S$ of finite index in $\bar{G}$ dividing $n \alpha$. Replacing $S$ by $\bar{S}$ if necessary we may assume that 
$G^{0} \subseteq S$. Then

$$
\frac{G \cap S}{G^{0}} \cong \frac{(G \cap S) G^{0}}{\bar{G}^{0}}=\frac{S}{\bar{G}^{0}}=\frac{(H \cap S) \bar{G}^{0}}{\bar{G}^{0}} \cong \frac{H \cap S}{H \cap S \cap \bar{G}^{0}}
$$

and $H \cap S$ is a finite abelian group of rank at most $n$. Define $T$ by

$$
\frac{T}{G^{0}}=\bigcap_{\phi \in \Delta u t\left(G / G^{0}\right)}\left(\frac{G \cap S}{G^{0}}\right) \varphi .
$$

Clearly $T$ has all the required properties except possibly the bound on the index. But if $K$ is any subgroup of $G$ with $G^{0} \subseteq K$ and $(G: K)$ dividing $n \alpha$ then $(G \cap S)^{n \alpha} G^{0} \subseteq K$, so $(G \cap S)^{n \alpha} G^{0} \subseteq T$. Since $(G \cap S) / G^{0}$ is finite abelian of rank at most $n$ the result follows.

The obvious analogue of Lemma 1 for fields of positive characteristic is false even for soluble groups $G$. If $r$ is any positive integer and $p$ any prime, it is easy to construct a linear group $G$ of degree $p+2$ over any non-algebraic field of characteristic $p$ that is the direct product of an elementary abelian $p$-group of rank $r$ and the wreath product of an infinite cycle by a cyclic group of order $p$, in which $G^{0}$ is the base group of the wreath product. Thus $G / G^{0}$ is characteristically simple, being elementary abelian of order $p^{r+1}$. If $G$ were triangularizable then $G$ would be abelian and so $G^{0}$ is the only triangularizable subgroup $T$ of $G$ containing $G^{0}$ with $T / G^{0}$ characteristic in $G / G^{0}$.

However, a modification of Lemma 1 does work for positive characteristics. For each positive integer $n$ let $n \mu$ be the least positive integer such that every soluble linear group of degree $n$ contains a triangularizable normal subgroup of finite index dividing $n \mu$. Such exists by Mal'cev's theorem.

Lemma 2. Let $G$ be a soluble linear group of degree $n$ and characteristic $p>0$. Then there exist subgroups $X, P$ and $Y$ of $G$ such that $(G: X)$ divides $(n \mu)^{n+1}$, $X^{p^{*}} \subseteq Y, X=P Y, G^{0} \subseteq P \cap Y, P / G^{0}$ and $Y / G^{0}$ are characteristic in $G / G^{0}, P / G^{0}$ is a p-group and $Y$ is triangularizable.

PRoof. We proceed as in the proof of Lemma 1, except that now $S$ is normal in $G$ with $(\bar{G}: S)$ dividing $n \mu$ and $H \cap S$ here is an extension of a $p$-group by a finite abelian $p^{\prime}$-group of rank at most $n$. Define $P$ and $Y$ by

$$
\frac{P}{G^{0}}=O_{p}\left(\frac{G}{G^{0}}\right) \text { and } \frac{Y}{G^{0}}=\bigcap_{\phi \in \operatorname{Aut}\left(G / G^{0}\right)}\left(\frac{G \cap S}{G^{0}}\right) \varphi \text {. }
$$

Since $G \cap S$ is normal in $G$ we have that $O_{p}\left((G \cap S) / G^{0}\right) \subseteq P / G^{0}$ and so $(G: X)$ divides $(n \mu)^{n+1}$. Also $P / G^{0}$ is isomorphic to a $p$-section of $H$ and thus its exponent divides $p^{n}$. Consequently $X^{p^{n}} \subseteq Y$. The remainder follows. 
RemarK. If in Lemma 2 the group $G$ is also finite, then $P=O_{p}(G)$ is unipotent and $X$ itself is triangularizable.

We require a slight extension of a lemma of Blichfeldt.

LEMMA 3. If $G$ is a linear group of degree $n$ and if $T$ is a triangularizable normal subgroup of $G$ then $\left(G: C_{G}(T / u(T))\right)$ divides $n !$.

Proof. Suppose $G \subseteq \mathrm{GL}(n, F)$ where $F$ is algebraically closed. There exist absolutely irreducible representations $\rho_{1}, \ldots, \rho_{r}$ say of $G$ and an $x \in \operatorname{GL}(n, F)$ such that

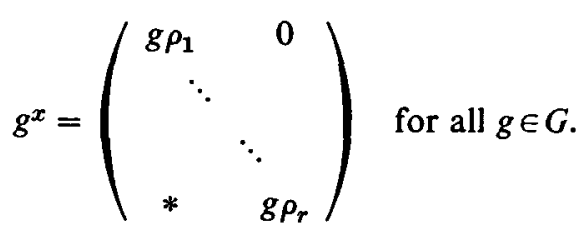

Let $\rho$ be the representation of $G$ given by $g \rho=\operatorname{diag}\left(g \rho_{1}, \ldots, g \rho_{r}\right)$ for each $g \in G$. Clearly $T \rho$ is triangularizable and by Clifford's Theorem $T \rho$ is completely reducible over $F$, so $T \rho$ is actually diagonalizable. Thus $\left(G \rho: C_{G \rho}(T \rho)\right)$ divides $n$ ! by Wehrfritz (1973), 1.12. But ker $\rho=u(G)$ and $T \cap u(G)=u(T)$. Thus $\left(G: C_{G}(T / u(T))\right)$ divides $n$ !.

LEMMA 4. Let $G$ be a soluble subgroup of $\mathrm{GL}(n, F)$. Then $G$ contains a triangularizable subgroup $T$ such that $G^{0} \subseteq T, T$ is normalized by $N_{\mathrm{Aut} G}\left(G^{0}\right)$ and $(G: T)$ divides $n !(n \mu)^{n+1}$.

The proof of Lemma 1 yields the characteristic zero case of Lemma 4 and actually produces a suitable subgroup $T$ with $(G: T)$ dividing $(n \mu)^{n+1}$. Since Lemma 1 already includes the characteristic zero case of Proposition 1 we ignore this case of Lemma 4 and assume in the proof below that char $F=p>0$. Note that this will complete the proof of Proposition 1.

Proof. We may assume that $F$ is algebraically closed. Again denote closures in GL $(n, F)$ by a bar. Choose $X$ and $Y$ as in Lemma 2 . Then $Y$ is a triangularizable normal subgroup of $\bar{G}$. Let $C=C_{\bar{X}}(\bar{Y} / u(\bar{Y}))$. Now $C=\bar{C}$ and so by Wehrfritz (1973), 10.10 again there exists a finite $p$-subgroup $U$ of $C$ such that $C=U \bar{Y}$. Since $U . u(C)$ is a p-group it is unipotent, and it is normal in $C$ since $C^{\prime} \subseteq U . u(C)$. Thus $U \cdot u(C) . \bar{Y}=C$ is triangularizable. 
Set $T=C_{X}(Y / u(Y))$. Since $u(Y)=O_{p}(Y)$ every automorphism of $G$ normalizing $G^{0}$ normalizes $T$. Also by Wehrfritz (1973), 5.10 we have

$$
[T, \bar{Y}] \subseteq \overline{u(Y)} \subseteq u(\bar{Y}),
$$

and so $T \subseteq C$ and $T$ is triangularizable. Finally $(X: T)$ divides $n$ ! by Lemma 3 and so $(G: T)$ divides $n !(n \mu)^{n+1}$.

Proof of Lemma 5. Let $E$ be a shallow field, $X$ a set of indeterminates over $E$, $F$ a finite extension of $K=E(X)$ and $L$ the splitting field over $F$ of some polynomial over $F$ of degree $n$. Then $[L: F] \leqslant n$ !. The roots of unity in $L$ lie in the algebraic closure $L_{0}$ of $E$ in $L$. Now $L_{0}$ and $K$ are linearly disjoint since $X$ is still algebraically independent over $L_{0}$. Hence $\left[L_{0}: E\right]=\left[L_{0}(X): K\right]$ which divides $[L: F][F: K]$, and since $E$ is shallow it follows that $F$ is shallow also.

Proof of Proposition 2. Let $G$ be a subgroup of $\operatorname{GL}(n, F)$ where the field $F$ is shallow at $n$ and denote by $F$ the algebraic closure of $F$. As in the proof of Lemma 1 , Platonov's lemma (Wehrfritz (1973), 10.10) implies there exist a finite subgroup $H$ of $\mathrm{GL}(n, F)$ with a normal subgroup $K$ such that $G / G^{0} \cong H / K$. Now for primes $q \neq \operatorname{char} F$ the Sylow $q$-subgroups of $H$ are monomial (Wehrfritz (1973), 1.14 and 1.6) and so contain an abelian normal subgroup of rank at most $n$ and index dividing $n$ !. If we can show that every element if $\operatorname{char} F=0$ and every $p^{\prime}$-element if $\operatorname{char} F=p>0$, of $G / G^{0}$, has order dividing $n \delta$ the proposition will follow.

Let $g \in G$. Then $\langle g\rangle^{0} \subseteq G^{0}$ and so we may assume that $G=\langle g\rangle$. Let $E$ be the subfield of $F$ generated by $F$ and the eigenvalues of $g$. By hypothesis every root of unity in $E$ has order dividing $n \delta$. Let $g=g_{u} g_{d}$ be the Jordan decomposition of $g$ in $\operatorname{GL}(n, F)$, cf. Wehrfritz (1973), Chapter 7. By the choice of $E$ actually $\mathrm{g}_{u}, g_{d} \in \mathrm{GL}(n, E)$ and some conjugate $d=\operatorname{diag}\left(d_{1}, \ldots, d_{n}\right)$ of $g_{d}$ in $\operatorname{GL}(n, E)$ is actually diagonal. Also by Wehrfritz (1973), 7.3 the group $G$ is dense in $\left\langle g_{u}, g_{d}\right\rangle$. Consequently $G^{0}=G \cap\left\langle g_{u}, g_{d}\right\rangle^{0}$.

If $\operatorname{char} F=0$ then $\left\langle g_{u}\right\rangle$ is connected and $g_{u} \in\left\langle g_{u}, g_{d}\right\rangle^{0}$. If $\left.\operatorname{char} F=p\right\rangle 0$ then $\left\langle g_{u}\right\rangle$ is a $p$-group. In either case $\left\langle g_{d}\right\rangle^{0} \subseteq\left\langle g_{u}, g_{d}\right\rangle^{0}$ and it suffices to prove that $\left(\left\langle g_{d}\right\rangle:\left\langle g_{d}\right\rangle^{0}\right)$ divides $n \delta$. This index of course is equal to $\left(\langle d\rangle:\langle d\rangle^{0}\right)$. Now, closed diagonal groups are determined by monomial equations (for example, Borel (1969), Section 8, especially 8.4). Thus there exists a finite set of integers $e_{j k}, j=1,2, \ldots, n$, and say $k=1,2, \ldots, r$ such that for each integer $i, d^{i} \in\langle d\rangle^{0}$ if and only if

$$
d_{1}^{i e_{1 k}} d_{2}^{i e_{2 k} \ldots} \ldots d_{n}^{i e_{n k}=1} \text { for } k=1,2, \ldots, r .
$$

Now $\left(\langle d\rangle:\langle d\rangle^{0}\right)$ is finite so there exists $i>0$ with $d^{i} \in\langle d\rangle^{0}$. Whence $d_{1}^{e_{1 k}} \ldots d_{n}^{e_{n k}}$ is a root of unity for each $k$, which by definition has order dividing $n \delta$. Thus $d^{n \delta} \in\langle d\rangle^{0}$ and the proof is complete. 
Proof of Corollary 2. Part (a) is immediate from Proposition 2 and the proof of Wehrfritz (1973), 4.3 yields (b). Suppose $G$ and $T$ are as in (c). From Lemma 3 we have that $\left(G^{+}: T\right)$ divides $n$ !, which with (a) yields the required bound on $(G: T)$. Finally that $T$ is triangularizable follows just as in the proof of Lemma 4 .

COROLLARY 3. Let $G$ be a subgroup of $\mathrm{GL}(n, F)$ where $F$ is shallow at $n$ and $H a$ closed subgroup of $G$. If $x$ is an element of $G$ such that $x^{m} \in H$, where $m$ is a positive integer, prime to char $F$ if this is positive, then $x^{n \delta} \in H$.

Proof. $H \cap\langle x\rangle$ is closed of finite index in $\langle x\rangle$, so $\langle x\rangle^{0} \subseteq H$. The result now follows from Proposition 2.

\section{References}

A. Borel (1969), Linear Algebraic Groups (Benjamin, New York).

K. W. Gruenberg (1968), "The hypercentre of linear groups", J. Algebra 8, 34-40.

B. A. F. Wehrfritz (1973), Infinite Linear Groups (Springer, Berlin-Heidelberg-New York).

B. A. F. Wehrfritz (1978), "Nilpotence in groups of semilinear maps I", Proc. London Math. Soc. (3) 36, 448-479.

Queen Mary College

London El 4NS

United Kingdom 